\title{
PA-134 NONTYPHOIDAL SALMONELLA IN THE FOODSTUFFS AND THE HUMAN DIARRHOEAL STOOLS IN OUAGADOUGOU, BURKINA FASO
}

Marguerite Nikiema, ${ }^{1}$ Absatou Ky-Ba, ${ }^{2}$ Kiswendsida Abdou Muller Comapore, ${ }^{2}$ Alfred Traore, ${ }^{3}$ Lassana Sangare ${ }^{1}$. ${ }^{1}$ CHUYO, Burkina Faso; ${ }^{2}$ Laboratoire National de Santé Publique, Burkina Faso; ${ }^{3}$ UFR-SVT, University of Ouagadougou, Burkina Faso

10.1136/bmjgh-2016-000260.160

Background The sanitary quality of food is a global concern. Salmonella infections are a major health problem in developing countries. Each year, food poisoning is affecting thousands of consumers. The objective of this study was to isolate strains of nontyphoidal Salmonella in food and in human diarrhoeal stools in Ouagadougou.

Methods Sixty-one samples of sandwiches bought in Ouagadougou and 177 diarrhoeic stools specimen collected at the University Hospital Yaldao Ouedraogo and the Medical Centre Schiphra from May to October 2015 to detect Salmonella. The antibiotic susceptibility testing of Salmonella strains was done by the disk diffusion method using 14 antibiotics. Statistical analysis of data was done with Epi Info 7.3.

Results From the overall samples analysed, 23 strains of Salmonella were identified including14/177 (7.9\%) clinical strains, 9/61 (14.75\%) food strains. After antigenic identification 15 isolates (6 from foods, 9 from stools) belonged to known serotypes including 9 typhoidal and 6 nontyphoidal stains. Eight strains (3 from foods, 5 from stools) could not be serotyped by the reagents available. All the serotypes identified were found in stools (2 S. typhi, 1 S. paratyphi B, $1 S$. paratyphi C, $1 \mathrm{~S}$. enteritidis, $3 \mathrm{~S}$. typhimurium and $1 \mathrm{~S}$. dublin) while $S$. paratyphi B (4), S. paratyphi C (1) and $S$. enteritidis (1) only were identified in foods. Eleven $(47.83 \%)$ strains were resistant to cotrimoxazole $(2 / 11)$, tetracycline $(8 /$ $11)$, nalidixic acid (\#\#) and ciprofloxacine (2/11).

Conclusions The overall frequency of Salmonella is higher in the foods than in the diarrhoeic stools. However, the serotype diversity of the clinical strains is more important than that of the food strains. The street sandwiches would not be the main sources of contamination by Salmonella. The high rate of the Salmonella resistance to antibiotics requires a more steady surveillance of the use of these antimicrobials. 